\title{
Dementia and Other Chronic Diseases in Older Adults in Havana and Matanzas: The 10/66 Study in Cuba
}

\author{
Juan de Jesús Llibre MD MPH PhD, Adolfo Valhuerdi MD MS, Marina Calvo MD MS, Rosa M. García MD MS, Milagros Guerra \\ MD MS, Tania Laucerique MD MS, Ana M. López MD MS, Juan Carlos Llibre MD MS, Lisseth Noriega MD, Isis Y. Sánchez MD \\ MS, Rudbeskia Porto MD MS, Francis Arencibia MS, Beatriz Marcheco MD PhD, Carmen Moreno MD
}

\begin{abstract}
INTRODUCTION Chronic non-communicable diseases are the leading cause of death worldwide, except in Sub-Saharan Africa. Nonetheless, one of these conditions, dementia, is the major contributor to disability-adjusted life years in people aged $\geq 60$ years. Few epidemiological studies exist of the prevalence and impact of dementia and selected chronic diseases in older adults in Latin America.
\end{abstract}

OBJECTIVE Describe prevalence of dementia, other chronic vascular diseases and cardiovascular risk factors, as well as resulting disabilities and care needs generated in adults aged $\geq 65$ years in Havana City and Matanzas provinces, Cuba.

METHODS The 10/66 study is a prospective longitudinal study involving a cohort of 3015 adults aged $\geq 65$ years in municipalities of Havana City and Matanzas provinces, divided into two phases: a cross-sectional door-to-door study conducted in 2003-2006, and a follow-up and assessment phase in 2007-2010. This article reports findings from the first phase. Hypertension diagnosis was based on criteria from the International Society for Hypertension; diabetes mellitus on American Diabetes Association criteria; stroke according to WHO definitions; and dementia according to criteria of the American Psychiatric Society's Diagnostic and Statistical Manual of Mental Disorders DSMIV and the 10/66 International Dementia Research Group. Ischemic heart disease was defined by self-report of previous physician diagnosis. Study variables included age, sex, educational level, substance use (alcohol, tobacco) and dietary habits. A structured physical and neurological exam, including blood pressure measurement, was performed on all participants. Laboratory tests included complete blood count, fasting blood glucose, total cholesterol and lipoprotein fractions, triglycerides and apolipoprotein E genotype. Prevalence and standardized morbidity ratios (crude and adjusted) were calculated for chronic diseases studied with 95\% confidence intervals, using a Poisson regression model and indirect standardization.

RESULTS The study assessed 2944 older adults (response rate $97.6 \%$ ) and found high prevalence of vascular risk factors and of chronic non-communicable diseases: hypertension $73.0 \%(95 \% \mathrm{Cl}$ 71.4-74.7), diabetes mellitus $24.8 \%$ (95\% Cl 22.9-26.5), ischemic heart disease $14.1 \%(95 \% \mathrm{Cl} 12.9-15.4)$, dementia $10.8 \%(95 \% \mathrm{Cl}$ 9.7-12.0) and stroke $7.8 \%(95 \% \mathrm{Cl} 6.9-8.8)$. The majority of participants $(85 \%)$ had more than one cardiovascular risk factor. The main cause of disability and dependency in the study population was dementia.

CONCLUSION The high prevalence of chronic diseases observed in the elderly - with the consequent morbidity, disability and dependency-highlights the need for prevention, early diagnosis and risk factor control, particularly given the demographic and epidemiologic transition faced by Cuba and other developing countries.

KEYWORDS Dementia, chronic disease, cardiovascular diseases, heart disease, stroke, diabetes mellitus, disability, hypertension, aging, prevalence, epidemiology, Cuba

\section{INTRODUCTION}

Population aging is occurring worldwide and faster than anticipated.[1] Populations are growing slowly, with the proportion of older people increasing as a result of declining fertility and replacement rates coupled with longer life expectancies. Latin America, China and India are experiencing an unprecedented acceleration in population aging. As in other developing countries, chronic noncommunicable diseases (NCDs) are becoming a bigger burden.

NCDs are the leading cause of death worldwide, except in SubSaharan Africa, and in 2005, $80 \%$ of the 35 million deaths from NCDs occurred in low- and middle-income countries.[2] In part, this is due to the fact that $60 \%$ of the world's older adults now live there, a figure expected to reach $80 \%$ by 2050 .

However, changes in risk factor patterns are also contributing to this picture. The third stage in the epidemiologic transition is exemplified in Latin America, where, along with increased life expectancy, fat-rich diets, smoking and physical inactivity have become more common. As a result, cardiovascular diseases have become the main population health problem, more so than in stage two regions-e.g., China and India-where risk factors are less prevalent, or in stage four regions-e.g., Europe-where public health policies have reduced risk exposure levels.[3] INTERHEART, a multinational, case-control study, hypothesizes that risk factors for myocardial infarction operate similarly in all regions of the world, including Latin America and China.[4]

Twice as many people die annually from NCDs as from infectious diseases-including HIVIAIDS, tuberculosis, and malaria-maternal and perinatal causes and nutritional deficiencies combined. Thus, 388 million people worldwide will die from one or more NCDs over the next decade.[5,6] However, $80 \%$ of premature deaths due to cardiovascular disease, stroke and diabetes mellitus could be prevented with healthy lifestyles, early diagnosis and drug therapy. $[7,8]$

Although there is growing interest in assigning higher priority to NCDs in national and global health strategies, mental illnesses in general and dementia in particular are absent from the policy agendas of most low- and middle-income countries.[9]

Cuba is a developing country of 11.9 million with health indicators comparable to those of developed countries and a rapidly aging population. By the year 2020, it is predicted that Cuba will have the highest proportion of older adults in Latin America, with $25 \%$ aged $\geq 60$ years.[10] Currently, NCDs—and within them, in order of decreasing frequency, ischemic heart disease, cancer and stroke-are the leading causes of death, together accounting for about $60 \%$ of all deaths.[11] 
Given the demographic and health transitions described, more current and detailed information on the epidemiology of NCDs in older adults is urgently needed. The aim of this study is to describe in a cohort of older adults the prevalence of chronic diseases-including dementia-and their risk factors, as well as the disability and dependence they engender. We have included dementia because, in addition to being an NCD, it is the major contributor $(11.2 \%)$ to disability-adjusted life years in people aged $\geq 60$ years, accounting for more than stroke (9.5\%), musculoskeletal diseases $(8.9 \%)$, cardiovascular diseases $(5.0 \%)$ or cancer $(2.4 \%)$.[3]

\section{METHODS}

The 10/66 Study is a population-based, door-to-door study, involving a total sample of 21,000 participants aged $\geq 65$ years from 11 countries: Brazil, Cuba, Mexico, Peru, Puerto Rico, the Dominican Republic and Venezuela in Latin America; China and India in Asia; and Nigeria and South Africa in Africa. Instruments used were validated in pilot research carried out simultaneously in 26 countries including Cuba.[12]

The study's methodology enables diagnosis of dementia and its subtypes, other mental and physical health conditions as well as description of anthropometric measures and socio-demographic characteristics. An extensive questionnaire was administered to each participant, with questions on NCDs and risk factors, disability and functional levels, health services utilization, care arrangements and caregiver strain. This was rounded out with physical and neurological exams, complementary tests, and an interview with a reliable informant.

In Cuba, the 10/66 Study is a prospective, longitudinal cohort study of 3015 adults aged $\geq 65$ years, 2100 in Havana City province and 915 in Matanzas province. Two phases were adopted: the first, a cross-sectional prevalence study carried out door-to-door from June 2003 through December 2006; and the second, a follow-up and assessment study of the cohort from 2007 through 2010. Details of the research protocol and methods have been published elsewhere.[13-15] This article reports results from the first phase.

Variables The following variables were included:

- Sociodemographic characteristics: age, sex and educational level.

- Self-reported NCDs, based on questions such as: "Has a doctor ever diagnosed you with cerebrovascular disease / heart attack / angina / diabetes?", as well as description of symptoms.

- Smoking habits described as: current smoker, ex-smoker, never smoked; type of tobacco used (cigarettes, cigars, pipe tobacco, chewing tobacco, snuff); average number of units used per day; age at onset and age of quitting, if applicable (the last two used to compute length of exposure)

- Alcohol consumption and units of alcohol ingested per week, by amount consumed weekly before and after age 65. The cut-off level for high-risk drinkers was 14 units per week for women and 21 units per week for men. Participants were asked, "Was there ever a time in your life when you would have said you were a heavy drinker? Have you ever had treatment or help for problems with alcoholism?"
- Mean systolic and diastolic blood pressure, measured sitting and standing on two separate occasions. Two methods were used to diagnose hypertension: self-report of physician diagnosis ("Have you ever been told you had high blood pressure? When were you first told? Did you start a treatment? Are you still being treated?"); and/or a blood pressure reading meeting criteria of the International Society for Hypertension (systolic pressure $\geq 140 \mathrm{~mm} \mathrm{Hg}$ and/or diastolic pressure $\geq 90 \mathrm{~mm} \mathrm{Hg}$ ).[15]

- Diagnosis of dementia followed the criteria and diagnostic algorithm of the 10/66 Study[12,13] involving: a) a structured clinical interview, the Geriatric Mental State and its computerized algorithm (AGECAT);[16] b) the battery of cognitive tests from the Community Screening Interview for Dementia (CSI 'D') COGSCORE;[17] c) the verbal fluency task and 10-word list learning task with delayed recall, developed by the European Consortium to Establish a Registry for Alzheimer's Disease (CERAD);[18] d) an interview with a reliable informant, the CSI'D' RELSCORE[17] for evidence of cognitive and functional decline; and, e) additional information on onset and course of dementia provided by the Program for Diagnosis of Dementia and its Subtypes, History, and Etiology.[19] Criteria of the DSM-IV and American Psychiatric Society were also used.[20]

- Stroke diagnosis, defined by the $\mathrm{WHO}$, based on information from the participant and a reliable informant about sudden or rapidly developing signs of focal or global neurological dysfunction lasting $>24$ hours with no apparent nonvascular cause (cranial trauma; neoplasia; coma attributable to metabolic disorder or electrolyte imbalance; vasculitis; central nervous system infection; or peripheral neuropathy).[21]

All individuals surveyed were given a structured physical and neurological exam for objective and quantitative measurement of focal signs, parkinsonism, ataxia, apraxia and primitive reflexes (NEUROEX)[22] to detect stroke symptoms and signs using the US National Institutes of Health Stroke Scale (NIHSS).[23]

If a participant had had an imaging study (computerized axial tomography or magnetic resonance imaging), a written report from the study was sought, including neuroimaging findings and time elapsed since the event that prompted the study.

- Diabetes mellitus, diagnosed in two ways: 1) self-report of physician diagnosis of diabetes ("Have you ever been told you have diabetes? When were you first told? Did you start treatment? Are you still being treated?"); and/or 2) fasting glucose of $\geq 7.0 \mathrm{mmol} / \mathrm{L}$ confirmed on two different days.[24]

- Central obesity, defined according to criteria of the Third Report of the Adult Treatment Panel of the National Cholesterol Education Program (NCEP-ATP III): abdominal circumference $>102 \mathrm{~cm}$ in men and $>89 \mathrm{~cm}$ in women.[25]

- Disability, determined using the short, 12-item version of the WHO Disability Assessment Schedule II (WHODAS-II), which assesses six domains: communication, mobility, self-care, interpersonal interaction, activities of daily living and social participation.[26] This version showed strong internal consistency in the validation conducted in Cuba and in various centers involved in the 10/66 Study.[27] The cut-off for severe disability was the $90^{\text {th }}$ percentile of sample's score distribution. 
- Physical impairment, assessed through self-report from a list of 11 commonly occurring limiting conditions:[13] paralysis, weakness or loss of limb; vision problems; gastrointestinal problems; arthritis or rheumatism; heart problems; hypertension; hearing loss or deafness; breathlessness; difficulty breathing or asthma; fainting orblackouts; skin disorders; persistent cough. Thesewere coded as present if they interfered with activities to any degree.

- Self-reported health status, based on the following question: "How would you describe your general health over the past 30 days?" (very good, good, fair, poor, very poor).

- Dependency, assessed with the following instruments:

- Gilleard et al.'s scale for caregiver time spent on elder care[28]

- Davis et al.'s Caregiver Activity Survey of time spent assisting elder with daily living activities.[29]

- Older adults were placed in three categories: dependent (care needed most of the time), occasionally dependent (care needed occasionally), and independent (care not needed).

Analysis Prevalence by sex and age group was calculated of hypertension, diabetes mellitus, ischemic heart disease, dementia, and stroke; as was prevalence of one or more cardiovascular risk factors (smoking, hypertension, diabetes and central obesity). Prevalence ratios with $95 \%$ confidence intervals for the effects of age in the presence of one or more cardiovascular risk factors were calculated using Poisson regression.[30]

Age- and sex-specific international prevalence comparisons were made for hypertension, ischemic heart disease, diabetes mellitus and stroke, using indirect age standardization and the standardized morbidity ratio (SMR) with $95 \%$ confidence intervals, using as reference populations the US National Health and Nutrition Examination Survey (NHANES)[31] and data provided by US National Heart Lung and Blood Institute.[32] The same approach was used for dementia, using as a reference EURODEM, a meta-analysis of 12 European studies.[33]

Population-attributable fractions for association between chronic disease and disability were calculated using the WHODAS-II score, estimating the proportion of disability avoidable if each health condition were eliminated, assuming causality and absence of confounding between health condition and outcome.

Poisson regression was used to evaluate associations between the various chronic diseases and dependency, estimating the population-attributable fraction for association between each chronic disease and dependency and between the combination of hypertension, diabetes and ischemic heart disease and dependency.

Data were entered into Epidata, exported to SPSS, and then to STATA version 9.2 for analysis.[34]

Ethical aspects Participants were asked for verbal and signed informed consent. If not capable of consent, permission was obtained from the responsible relative or guardian; confidentiality was protected of all information obtained in interviews. The study protocol was approved by the ethics committee of the Medical University of Havana.

\section{RESULTS}

Of 3015 older adults enumerated, 2944 interviews were completed, for a response rate of $97.6 \%$ overall, $97.3 \%(2043 / 2100)$ in Havana and 98.5\% (901/915) in Matanzas. Data were missing for some variables, hence variations reported in tables.

Sociodemographic characteristics The samples from Havana City and Matanzas were similar in sociodemographic characteristics, health profiles and behavior. The mean age of the study population was 74 years and over one quarter were aged $\geq 80$ years; $64.7 \%$ were women; $75 \%$ had completed primary school or beyond; only $9 \%$ of participants lived alone (Table 1 ).

Risk factors One-fifth of those surveyed were current smokers and approximately $90 \%$ did not consume alcohol; $7.5 \%$ were classified as high-risk drinkers before age 65 and $3.6 \%$ were still in this category. Only $6.4 \%$ perceived their health status as poor,

Table 1: Sociodemographic and health characteristics in selected populations: the 10/66 Study in Cuba

\begin{tabular}{|c|c|c|c|}
\hline & $\begin{array}{l}\text { Havana City } \\
\text { Province } \\
(n=2043) \\
\end{array}$ & $\begin{array}{c}\text { Matanzas } \\
\text { Province } \\
(\mathrm{n}=901) \\
\end{array}$ & $\begin{array}{c}\text { Total } \\
(n=2944)\end{array}$ \\
\hline & $n(\%)$ & n (\%) & n (\%) \\
\hline $\begin{array}{l}\text { Sex } \\
\text { Female } \\
\text { Male }\end{array}$ & $\begin{array}{r}1349(66.0) \\
694(34.0)\end{array}$ & $\begin{array}{l}555(61.6) \\
346(38.4)\end{array}$ & $\begin{array}{l}1904(64.7) \\
1040(35.3)\end{array}$ \\
\hline $\begin{array}{l}\text { Age group (years) } \\
65-69 \\
70-74 \\
75-79 \\
\geq 80 \\
\text { Unknown }\end{array}$ & $\begin{array}{r}542(26.5) \\
566(27.7) \\
445(21.8) \\
483(23.6) \\
7(0.3)\end{array}$ & $\begin{array}{r}218(24.2) \\
223(24.8) \\
194(21.5) \\
266(29.5) \\
0(0.0)\end{array}$ & $\begin{array}{r}760(25.8) \\
789(26.8) \\
639(21.7) \\
749(25.4) \\
7(0.2)\end{array}$ \\
\hline Living alone & $183(9.0)$ & $78(8.7)$ & 261 (8.9) \\
\hline $\begin{array}{l}\text { Marital status } \\
\text { Married } \\
\text { Widowed } \\
\text { Separate or divorced } \\
\text { Never married }\end{array}$ & $\begin{array}{r}876(43.0) \\
631(30.9) \\
357(17.5) \\
172(8.4)\end{array}$ & $\begin{array}{l}395(43.8) \\
297(33.0) \\
105(11.7) \\
103(11.4)\end{array}$ & $\begin{array}{r}1271(43.2) \\
928(31.5) \\
462(15.7) \\
275(9.3)\end{array}$ \\
\hline $\begin{array}{l}\text { Education } \\
\text { None } \\
\text { Incomplete primary } \\
\text { Primary completed } \\
\text { Secondary completed } \\
\text { University completed }\end{array}$ & $\begin{array}{r}49(2.4) \\
458(22.4) \\
661(32.4) \\
469(23.0) \\
398(19.5)\end{array}$ & $\begin{array}{r}26(2.9) \\
197(21.9) \\
318(35.3) \\
259(28.7) \\
101(11.2)\end{array}$ & $\begin{array}{r}75(2.5) \\
655(22.2) \\
979(33.3) \\
728(24.7) \\
499(16.9)\end{array}$ \\
\hline Physical inactivity & $629(30.8)$ & $324(36.0)$ & $953(32.4)$ \\
\hline $\begin{array}{l}\text { Smoking } \\
\text { Never smoked } \\
\text { Ex-smoker } \\
\text { Currently smokes }\end{array}$ & $\begin{array}{r}1125(55.1) \\
513(25.1) \\
403(19.7)\end{array}$ & $\begin{array}{l}490(54.4) \\
251(27.9) \\
162(18.0)\end{array}$ & $\begin{array}{r}1615(54.9) \\
764(26.0) \\
565(19.2)\end{array}$ \\
\hline $\begin{array}{l}\text { Alcohol use } \\
\text { Abstainer } \\
\text { High-risk drinker }\end{array}$ & $\begin{array}{r}1754(85.9) \\
77(3.8)\end{array}$ & $\begin{array}{r}785(87.1) \\
28(3.1)\end{array}$ & $\begin{array}{r}2539(86.2) \\
105(3.6)\end{array}$ \\
\hline $\begin{array}{l}\geq 3 \text { limiting } \\
\text { conditions }\end{array}$ & $201(9.8)$ & $91(10.1)$ & $292(9.9)$ \\
\hline $\begin{array}{l}\text { Poor perceived } \\
\text { health }\end{array}$ & $151(7.4)$ & $37(4.1)$ & $188(6.4)$ \\
\hline $\begin{array}{l}\text { Risk factors } \\
\text { None } \\
\text { One } \\
\text { Two } \\
\text { Three or more }\end{array}$ & $\begin{array}{l}282(13.8) \\
767(37.5) \\
685(33.5) \\
292(14.3)\end{array}$ & $\begin{array}{r}135(15.0) \\
375(41.6) \\
323(35.8) \\
85(9.4)\end{array}$ & $\begin{array}{r}417(14.2) \\
1142(38.8) \\
1008(34.2) \\
377(12.8)\end{array}$ \\
\hline
\end{tabular}

a Smoking, hypertension, diabetes, obesity 
the percentage being higher in Havana City than in Matanzas. More than $80 \%$ of those interviewed had one or more risk factors (smoking, hypertension, diabetes, or central obesity), including those without previous cardiovascular disease. Frequencies of the selected risk factors and prevalence ratios by age groups are shown in Table 2.

Over $85 \%$ of these adults aged $\geq 65$ years exhibited cardiovascular risk factors, a very high prevalence; and over half of them did, even when hypertension was excluded. Prevalence of having one or more risk factors declined in those aged $\geq 75$ years ( $p$ value for trend <0.001), except in the group aged 75-79 years when hypertension was excluded as a risk factor (Table 2).
NCDs Elevated prevalence of NCDs was found, in order of decreasing frequency: hypertension $73.0 \%$, diabetes mellitus $24.8 \%$, ischemic heart disease $14.1 \%$, dementia $10.8 \%$ and stroke $7.8 \%$. All these conditions except stroke were more prevalent in women in all age groups; stroke showed higher prevalence in men in all age groups (Table 3 ).

Subsequent to indirect standardization, the standardized morbidity ratio was obtained for hypertension, ischemic heart disease, diabetes mellitus, stroke, and dementia. A standardized morbidity ratio (SMR) of 100 implies that NCD prevalence in our study is similar to that found in the reference population; an SMR of less than 100 implies that prevalence is lower than that found in the reference population, and an SMR greater than

Table 2: Prevalence of $\geq 1$ risk factor by age and sex: the 10/66 Study in Cuba

\begin{tabular}{|c|c|c|c|c|c|c|c|}
\hline \multirow{3}{*}{ Risk Factors } & \multirow{3}{*}{ Sex } & \multicolumn{5}{|c|}{ Age group (years) } & \multirow[b]{2}{*}{$\begin{array}{r}\text { Overall } \\
\text { prevalence (\%) }\end{array}$} \\
\hline & & $\begin{array}{r}65-69 \\
(n=760)\end{array}$ & $\begin{array}{r}70-74 \\
(n=780)\end{array}$ & $\begin{array}{r}75-79 \\
(n=639)\end{array}$ & $\begin{array}{r}80+ \\
(n=749)\end{array}$ & $\begin{array}{r}\text { All Ages } \\
(n=2928\end{array}$ & \\
\hline & & $\begin{array}{r}\text { Prevalence ( } \%) \\
(95 \% \mathrm{Cl})\end{array}$ & $\begin{array}{r}\text { Prevalence }(\%) \\
(95 \% \mathrm{Cl})\end{array}$ & $\begin{array}{r}\text { Prevalence (\%) } \\
(95 \% \mathrm{Cl})\end{array}$ & $\begin{array}{r}\text { Prevalence }(\%) \\
(95 \% \mathrm{CI})\end{array}$ & $\begin{array}{r}\text { Prevalence (\%) } \\
(95 \% \mathrm{CI})\end{array}$ & $\begin{array}{r}\text { Prevalence }(\%) \\
(95 \% \mathrm{Cl})\end{array}$ \\
\hline \multirow{3}{*}{$\geq$ Risk Factor ${ }^{a}$} & $\mathrm{~F}$ & $\begin{array}{r}87.9 \\
(85.0-90.8)\end{array}$ & $\begin{array}{r}91.1 \\
(88.6-93.6)\end{array}$ & $\begin{array}{r}86.3 \\
(83.0-89.6)\end{array}$ & $\begin{array}{r}82.9 \\
(79.7-86.2)\end{array}$ & $\begin{array}{r}87.0 \\
(85.5-88.5)\end{array}$ & \multirow{3}{*}{$\begin{array}{r}85.8 \\
(84.8-87.0)\end{array}$} \\
\hline & M & $\begin{array}{r}86.4 \\
(82.4-90.5)\end{array}$ & $\begin{array}{r}87.0 \\
(83.1-90.9)\end{array}$ & $\begin{array}{r}81.7 \\
(76.7-86.7)\end{array}$ & $\begin{array}{r}78.1 \\
(72.8-83.4)\end{array}$ & $\begin{array}{r}83.6 \\
(81.3-85.8)\end{array}$ & \\
\hline & $\mathrm{PR}^{\mathrm{b}}$ & $($ ref. $=1.00)$ & $\begin{array}{r}0.99 \\
(0.97-1.03)\end{array}$ & $\begin{array}{r}0.96 \\
(0.93-0.99)\end{array}$ & $\begin{array}{r}0.93 \\
(0.90-0.91)\end{array}$ & $\begin{array}{r}0.97 \\
(0.96-0.99)^{c}\end{array}$ & \\
\hline \multirow{3}{*}{$\begin{array}{l}\geq \text { Risk Factor } \\
\text { excluding } \mathrm{HT}\end{array}$} & $\mathrm{F}$ & $\begin{array}{r}67.1 \\
(63.0-71.3)\end{array}$ & $\begin{array}{r}52.7 \\
(52.4-62.0)\end{array}$ & $\begin{array}{r}58.2 \\
(52.4-64.1)\end{array}$ & $\begin{array}{r}50.9 \\
(44.9-57.3)\end{array}$ & $\begin{array}{r}60.8 \\
(58.7-63.0)\end{array}$ & \multirow{2}{*}{$\begin{array}{r}57.3 \\
(55.5-59.1)\end{array}$} \\
\hline & $\mathrm{M}$ & $\begin{array}{r}67.8 \\
(63.7-71.9)\end{array}$ & $\begin{array}{r}51.2 \\
(46.8-55.5)\end{array}$ & $\begin{array}{r}53.4 \\
(47.7-59.2)\end{array}$ & $\begin{array}{r}38.6 \\
(33.3-44.9)\end{array}$ & $\begin{array}{r}50.8 \\
(47.8-53.9)\end{array}$ & \\
\hline & $\mathrm{PR}^{\mathrm{b}}$ & $($ ref. $=1.00)$ & $\begin{array}{r}0.98 \\
(0.90-1.07)\end{array}$ & $\begin{array}{r}0.86 \\
(0.78-0.95)\end{array}$ & $\begin{array}{r}0.70 \\
(0.63-0.78)\end{array}$ & $\begin{array}{r}0.89 \\
(0.86-0.92)^{c}\end{array}$ & \\
\hline
\end{tabular}

aAny of: smoking, hypertension, diabetes, central obesity

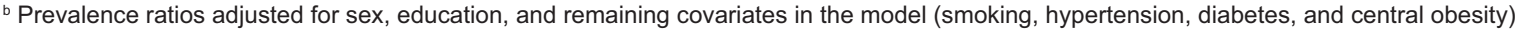

${ }^{c} \mathrm{p}$ value for trend $<0.001$

Table 3: Prevalence of selected chronic diseases in Cuban population samples and international comparisons: the 10/66 Study in Cuba

\begin{tabular}{|c|c|c|c|c|c|c|c|c|}
\hline & \multicolumn{7}{|c|}{ Age (years) } & \multirow[b]{2}{*}{$\begin{array}{l}\text { SMR adjusted } \\
\text { for age and } \\
\text { sex }(95 \% \mathrm{CI})\end{array}$} \\
\hline & Sex & $\begin{array}{r}65-69 \\
(n=760)\end{array}$ & $\begin{array}{r}70-74 \\
(n=780)\end{array}$ & $\begin{array}{r}75-79 \\
(n=639)\end{array}$ & $\begin{array}{r}80+ \\
(n=749)\end{array}$ & $\begin{array}{r}\text { All ages } \\
(n=2928)\end{array}$ & $\begin{array}{r}\text { Overall } \\
\text { prevalence }(\%)\end{array}$ & \\
\hline \multirow{2}{*}{ Hypertension } & $\mathrm{F}$ & $\begin{array}{r}70.4 \\
(66.4-74.5)\end{array}$ & $\begin{array}{r}80.0 \\
(76.5-83.7)\end{array}$ & $\begin{array}{r}76.0 \\
(71.9-80.1)\end{array}$ & $\begin{array}{r}73.4 \\
(69.6-77.2)\end{array}$ & $\begin{array}{r}75.0 \\
(73.0-76.9)\end{array}$ & \multirow{2}{*}{$\begin{array}{r}73.0 \\
(71.4-74.7)\end{array}$} & \multirow{2}{*}{$\begin{array}{r}104^{\mathrm{a}} \\
(100-109)\end{array}$} \\
\hline & M & $\begin{array}{r}71.4 \\
(66.1-76.8)\end{array}$ & $\begin{array}{r}70.9 \\
(65.7-76.1)\end{array}$ & $\begin{array}{r}67.8 \\
(61.8-73.9)\end{array}$ & $\begin{array}{r}66.1 \\
(60.0-72.2)\end{array}$ & $\begin{array}{r}69.2 \\
(66.3-71.9)\end{array}$ & & \\
\hline \multirow{2}{*}{ Stroke } & $\mathrm{F}$ & $\begin{array}{r}4.6 \\
(2.7-6.4)\end{array}$ & $\begin{array}{r}6.7 \\
(4.5-8.8)\end{array}$ & $\begin{array}{r}7.1 \\
(4.6-9.6)\end{array}$ & $\begin{array}{r}9.4 \\
(6.9-12.0)\end{array}$ & $\begin{array}{r}7.0 \\
(5.8-8.1)\end{array}$ & \multirow{2}{*}{$\begin{array}{r}7.8 \\
(6.9-8.8)\end{array}$} & \multirow{2}{*}{$\begin{array}{r}88^{b} \\
(74-96)\end{array}$} \\
\hline & M & $\begin{array}{r}6.3 \\
(3.4-9.2)\end{array}$ & $\begin{array}{r}9.2 \\
(5.9-12.2)\end{array}$ & $\begin{array}{r}8.0 \\
(4.4-11.6)\end{array}$ & $\begin{array}{r}15.1 \\
(10.4-19.7)\end{array}$ & $\begin{array}{r}9.5 \\
(7.7-11.3)\end{array}$ & & \\
\hline \multirow{2}{*}{ Dementia } & $\mathrm{F}$ & $\begin{array}{r}2.9 \\
(1.4-4.4)\end{array}$ & $\begin{array}{r}6.3 \\
(4.1-8.4)\end{array}$ & $\begin{array}{r}9.8 \\
(6.9-12.6)\end{array}$ & $\begin{array}{r}26.8 \\
(23.0-30.7)\end{array}$ & $\begin{array}{r}11.6 \\
(10.3-13.1)\end{array}$ & \multirow{2}{*}{$\begin{array}{r}10.8 \\
(9.7-12.0)\end{array}$} & \multirow{2}{*}{$\begin{array}{r}116^{c} \\
(105-122)\end{array}$} \\
\hline & M & $\begin{array}{r}2.8 \\
(1.0-4.9)\end{array}$ & $\begin{array}{r}6.1 \\
(3.4-8.9)\end{array}$ & $\begin{array}{r}7.0 \\
(3.7-10.4)\end{array}$ & $\begin{array}{r}23.3 \\
(17.8-28.7)\end{array}$ & $\begin{array}{r}9.2 \\
(7.6-11.1)\end{array}$ & & \\
\hline \multirow{2}{*}{ Diabetes } & $F$ & $\begin{array}{r}26.3 \\
(21.9-30.6)\end{array}$ & $\begin{array}{r}30.0 \\
(25.6-34.5)\end{array}$ & $\begin{array}{r}29.2 \\
(24.2-34.2)\end{array}$ & $\begin{array}{r}24.8 \\
(20.5-29.1)\end{array}$ & $\begin{array}{r}27.5 \\
(25.2-29.8)\end{array}$ & \multirow{2}{*}{$\begin{array}{r}24.8 \\
(22.9-26.5)\end{array}$} & \multirow{2}{*}{$\begin{array}{r}117^{\mathrm{a}} \\
(107-126)\end{array}$} \\
\hline & M & $\begin{array}{r}19.6 \\
(14.1-25.0)\end{array}$ & $\begin{array}{r}22.2 \\
(16.9-27.6)\end{array}$ & $\begin{array}{r}18.1 \\
(12.2-23.9)\end{array}$ & $\begin{array}{r}17.2 \\
(11.7-22.7)\end{array}$ & $\begin{array}{r}19.4 \\
(16.6-22.2)\end{array}$ & & \\
\hline \multirow{2}{*}{$\begin{array}{l}\text { Ischemic Heart } \\
\text { Disease }\end{array}$} & $\mathrm{F}$ & $\begin{array}{r}12.6 \\
(9.8-15.7)\end{array}$ & $\begin{array}{r}15.1 \\
(12.0-18.3)\end{array}$ & $\begin{array}{r}15.6 \\
(12.1-19.2)\end{array}$ & $\begin{array}{r}14.8 \\
(11.7-17.9)\end{array}$ & $\begin{array}{r}14.5 \\
(12.9-16.1)\end{array}$ & \multirow{2}{*}{$\begin{array}{r}14.1 \\
(12.9-15.4)\end{array}$} & \multirow{2}{*}{$\begin{array}{r}106^{\mathrm{a}} \\
(101-112)\end{array}$} \\
\hline & M & $\begin{array}{r}12.8 \\
(8.8-16.8)\end{array}$ & $\begin{array}{r}13.4 \\
(9.5-17.2)\end{array}$ & $\begin{array}{r}15.3 \\
(10.7-20.0)\end{array}$ & $\begin{array}{r}12.4 \\
(8.2-16.7)\end{array}$ & $\begin{array}{r}13.4 \\
(11.3-15.5)\end{array}$ & & \\
\hline
\end{tabular}

a US National Health and Nutrition Examination Survey (=100, reference)[31]

${ }^{b}$ US National Heart Lung and Blood Institute (=100, reference)[32]

${ }^{c}$ EURODEM (=100, reference)[33] 
Table 4: Prevalence of chronic diseases in older adults and contribution to disability and dependency: the 10/66 Study in Cuba

\begin{tabular}{|c|c|c|c|c|c|c|}
\hline \multirow[b]{2}{*}{ Chronic Disease } & \multirow{2}{*}{$\begin{array}{l}\text { Prevalence } \\
\text { (CI 95\%) }\end{array}$} & \multicolumn{2}{|c|}{ Disability } & \multicolumn{3}{|c|}{ Dependency } \\
\hline & & $\begin{array}{r}R^{a} \\
(95 \% \mathrm{Cl})\end{array}$ & $\begin{array}{r}\text { PAF }^{\mathrm{b}} \\
(\%)\end{array}$ & $\begin{array}{r}\text { Rank order } \\
\text { (by PAF) }\end{array}$ & $\begin{array}{r}\mathrm{RR}^{\mathrm{c}} \\
(95 \% \mathrm{Cl})\end{array}$ & $\begin{array}{r}\text { PAF }^{d} \\
(\%)\end{array}$ \\
\hline Dementia & $\begin{array}{r}10.8 \\
(9.7-12.0)\end{array}$ & $\begin{array}{r}2.44 \\
(2.19-2.71)\end{array}$ & 43.6 & 1 & $\begin{array}{r}17.3 \\
(11.2-26.7)\end{array}$ & 64.6 \\
\hline Stroke & $\begin{array}{r}7.8 \\
(6.9-8.8)\end{array}$ & $\begin{array}{r}1.38 \\
(1.23-1.55)\end{array}$ & 11.4 & 2 & $\begin{array}{r}2.5 \\
(1.9-3.4)\end{array}$ & 23 \\
\hline Ischemic Heart Disease & $\begin{array}{r}14.1 \\
(12.9-15.4)\end{array}$ & $\begin{array}{r}1.07 \\
(0.95-1.21)\end{array}$ & 3.1 & 3 & & \\
\hline Diabetes & $\begin{array}{r}24.8 \\
(22.9-26.5)\end{array}$ & $\begin{array}{r}1.10 \\
(1.01-1.16)\end{array}$ & 2.5 & 4 & $\begin{array}{r}1.5 \\
(1.2-1.8)^{\mathrm{e}}\end{array}$ & 3. \\
\hline Hypertension & $\begin{array}{r}73.0 \\
(71.4-74.7)\end{array}$ & $\begin{array}{r}0.99 \\
(0.91-1.08)\end{array}$ & 2.1 & 5 & & \\
\hline
\end{tabular}

a Relative risk (estimated by prevalence ratio) of disability given chronic disease

${ }^{\mathrm{b}}$ Population attributable fraction for contribution of chronic disease to disability

${ }^{c}$ Relative risk (estimated by prevalence ratio) of dependency given chronic disease

${ }^{\mathrm{d}}$ Population attributable fraction for contribution of chronic disease to dependency

${ }^{e}$ Relative risk (estimated by prevalence ratio) of dependency given combination of hypertension,

diabetes and ischemic heart disease
City were interviewed. In this study, $44 \%$ of the older adults reported having hypertension, a majority of them women, and $14.8 \%$ of participants reported a history of diabetes mellitus. The higher prevalences of hypertension and diabetes mellitus found in our study may relate to the fact that SABE used only self reports, while we combined self reporting with blood pressure and fasting blood glucose measurements. The prevalence of stroke based on self reports was $9.7 \%$ in $\mathrm{Ha}$ vana City in SABE; we found a slightly lower prevalence of $7.8 \%$ using WHO criteria.[21]

The prevalence of hypertension in the population studied is as high as that reported in the United States. US NHANES (1999-2004) found a hypertension prevalence of $60 \%$ among those surveyed aged 60-69 years, increasing to $72 \%$ in those aged $70-79$ years and to $77 \%$ in those aged $\geq 80$ years.[31]
100 implies that prevalence is higher than that of the reference population.

Using US NHANES as a reference population, our study's SMR was elevated for diabetes: SMR 117 (95\% CI SMR 107-126); ischemic heart disease: SMR 106 (95\% Cl 101-112); and hypertension: SMR 104 (95\% Cl 100-109); and was reduced for stroke: SMR 88 (95\% Cl 74-96). For dementia, the prevalence rates by age and sex were higher than those reported by EURODEM: SMR 116 (95\% Cl 105-122).

Disability and dependency Table 4 shows prevalence of the chronic diseases studied and the contributions of these to disability and dependency, demonstrated by prevalence ratios and population attributable fractions. A strong association was found between risk of severe disability and dementia and stroke in older adults. The highest population attributable fraction for disability resulted from dementia, $43.6 \%$; the second highest was for stroke.

A participant with dementia was 17 times more likely than those without to need care most of the time (Table 4). In the case of people with stroke, need for such care was 2.5 times more likely. For hypertension, ischemic heart disease and diabetes mellitus, the probability was 1.5 times higher than in those who did not present with any of these conditions. Population attributable fractions for care needs were $64.6 \%$ for dementia, $23.1 \%$ for stroke, and $3.1 \%$ for the three other chronic diseases combined.

\section{DISCUSSION}

The study presents evidence of a high prevalence of NCDs and their vascular risk factors in older adults in Havana City and Matanzas. The prevalence of hypertension, diabetes, ischemic heart disease, and dementia was higher than reported for the same diseases in two studies used as references performed in developed countries: US NHANES[31] and EURODEM.[33]

Salud, Bienestar y Envejecimiento en las Américas (Health, Welfare, and Aging in the Americas, known by its Spanish acronym, SABE),[35] was a door-to-door, cross-sectional study carried out in the year 2000, in which 1905 people aged $\geq 60$ years in Havana
Numerous studies have demonstrated that adequate treatment and control of hypertension reduce cardiovascular morbidity and mortality in patients aged $60-80$ years.[36,37] Given that the absolute risk of experiencing a cardiovascular event is much greater in older people, the number of people with hypertension who need to be treated to prevent an adverse event is smaller in older adults: 22 in a 5-year period to prevent stroke and 45 to prevent a cardiovascular episode, compared with 113 to prevent stroke and 180 to prevent a cardiovascular episode in those aged $<65$ years.[38] Despite this evidence, available studies unfortunately indicate that knowledge, treatment and control of hypertension is inadequate.

Diabetes prevalence in our study is slightly higher than that reported in US NHANES 1999-2002,[39] in which prevalence increased with age, from $2.4 \%$ in those aged $20-39$ years to $21.6 \%$ in people aged $\geq 65$ years; overall diabetes prevalence increased slightly from $5.1 \%$ in $1988-1994$ to $6.5 \%$ in $1999-2002$, with greatest increases occurring in the elderly.[39] With population aging and increased prevalence of obesity, diabetes prevalence is expected to double by 2030.[24]

Self-reported diagnosis of ischemic heart disease in our study was confirmed by medical specialists; prevalence was higher than symptomatic ischemic heart disease prevalence reported in those aged $\geq 65$ years by US. NHANES.[31] By the year 2020, cardiovascular diseases-and first among them, arteriosclerosis-are projected to be the leading causes of worldwide disease burden, as measured by disability-adjusted life years.[40]

Stroke represents the third leading cause of death and the second cause of disability and dementia in the adult population over 65 worldwide. Stroke prevalence in our participants was lower than in the same age group reported by US National Heart, Lung and Blood Institute,[32] similar to that in an urban area of Madrid, 8.5\% (95\% Cl 5.5-11.5)[41] and higher than that reported in other Latin American studies.[42] In a recent publication,[43] we reported association of stroke in older Cuban adults with various risk factors, among them: hypertension, low HDL cholesterol, male sex, anemia, history of ischemic heart disease, carriage of one or two alleles of the apolipoprotein E (APOE) genotype, and older age. 
Dementia has become one of public health's most important problems, as the leading cause of disability and major contributor to dependency in older adults in Cuba and developed countries. $[13,44]$ Recent systematic reviews of prevalence data and expert consensus estimate that 36 million people suffer from dementia globally, with 4.6 million new cases annually (similar to the annual incidence of non-fatal stroke).[45,46] Dementia prevalence in this study, using 10/66 criteria, was higher than that reported in EURODEM. We have demonstrated that, at least for Cuba, 10/66 dementia criteria coincide better with specialists' clinical diagnosis than do DSM-IV criteria, which exclude a high proportion of mild dementia cases.[47] The Playa Study on Dementia and Alzheimer's (EDAP, its Spanish acronym) was a cross-sectional, door-to-door study carried out in 2002 of 18,000 people aged $\geq 65$ years in Playa municipality, Havana City province. EDAP found dementia in $9.3 \%$ of older adults, most female. Prevalence progressively increased with age. Education and cognitive training had a protective effect.[48]

Globally, cardiovascular diseases $(32.9 \%$ of years of potential life lost) and cancer $(22.5 \%$ of years of potential life lost) constitute the leading causes of premature death. However, they occupy eighth and ninth places among leading causes of disability, while dementia is the second leading cause of disability among chronic conditions in the elderly.[46] Dementia is not a simple unavoidable fact of aging but the final result of many risk factors and chronic vascular diseases-therefore preventable in a many cases.

A cohort effect as a result of the epidemiologic transition and increases in risk factors in the older adult population could explain the higher prevalence of NCDs reported in this study. The populationbased Cardiovascular Risk Factor Multiple Evaluation (CARMELA), a study of seven Latin American cities,[49] reported high frequency of cardiovascular risk factors, including obesity prevalence of $23 \%$ and smoking prevalence of 30\% among those aged 25-64 years.

The much greater association between dementia and both disability and dependency compared to the remaining NCDs studied is indicative of the magnitude of this growing health problem's impact on individuals, families and society. The observed population attributable fractions indicate that assuming a causal relationship between dementia and disability, if dementia did not exist as a health problem, the prevalence of severe disability would diminish by almost half in adults aged $\geq 65$ years.
Our research was based on a single-phase, door-to-door study, with a high response rate, and addressed prevalence of NCDs and risk factors as well as their impacts on disability and dependency in older adults. One limitation is that because they are local estimates, they cannot be generalized to the whole country.

Nonetheless, the strategy of surveying the entire population within selected areas facilitated longitudinal followup. Surveying the selected population in a single phase with a standardized questionnaire applied by specialized personnel, as described here, is a strength of the study. The questionnaire allowed us to obtain detailed information related to participants' physical and mental health, risk factors and care needs, as well as impact on family and community.

Cross-sectional studies provide local estimates of the prevalence of given diseases and health conditions needed for setting priorities among health conditions, developing health policies and strategies and planning service improvements. However, such studies need to be followed up to elucidate causal associations and assess changes in risk factor prevalence, disease prevalence and incidence, disability and dependency. Longitudinal studies are indispensable for exploring these additional dimensions; results from our 2007-2010 followup will provide further understanding of the dynamic of chronic vascular diseases in older adults and enable confirmation of associations observed in the first, crosssectional phase.

\section{CONCLUSIONS}

In view of the challenges presented by the demographic and epidemiologic transitions in developing countries, the high prevalence of NCDs in the elderly and their consequent morbidity, disability and dependence highlight the need for prevention, early diagnosis and risk factor control.

\section{ACKNOWLEDGMENTS}

This study is part of the 10/66 Dementia Research Group population-based research program in Cuba a collaborative agreement between the London Institute of Psychiatry and the Medical University of Havana sponsored by Alzheimer's Disease International, Wellcome Trust (GR066133), and the Cuban Ministry of Public Health. We are grateful to all those who participated in this population study. -1 -

\section{REFERENCES}

1. World Population Prospects: The 2002 Revision -Highlights. 2003. New York: United Nations; 2003 Feb 26. 22 p.

2. Fuster V, Voûte J. MDGs: chronic diseases are not on the agenda. Lancet. 2005 Oct29-Nov 4;366(9496):1512-4.

3. Yusuf S, Reddy S, Ounpuu S, Anand S. Global burden of cardiovascular diseases. Part I: General Considerations, the Epidemiologic Transition, Risk Factors, and Impact of Urbanization. Circulation. 2001 Nov 27;104(22):2746-53.

4. Yusuf S, Hawken S, Ounpuu S, Dans T, Avezum $A$, Lanas $F$, et al. Effect of potentially modifiable risk factors associated with myocardial infarction in 52 countries (the INTERHEART study): case-control study. Lancet. 2004 Sep 11-17;364(9438):937-52.

5. Lopez AD, Mathers CD, Ezzati M, Jamison DT, Murray CJL, editors. Global Burden of Disease and Risk Factors. Washington DC: Oxford Univ. Press; 2006. 506 p.

6. Mathers CD, Loncar D. Projections of global mortality and burden of disease from 2002 to 2030. PLoS Med. 2006 Nov;3(11):e442.

7. Strong K, Mathers C, Leeder S, Beaglehole R. Preventing chronic diseases: how many lives can we save? Lancet. 2005 Oct 29-Nov 4;366(9496):1578-82.

8. Alwan A, MacLean D, Riley LM, d'Espaignet ET, Mathers CD, Stevens GA, et al. Chronic Diseases and Development. Monitoring and surveillance of chronic non-communicable diseases: progress and capacity in highburden countries. Lancet. 2010 Nov 27;376(9755):1861-8.

9. Beaglehole R, Horton R. Chronic diseases: global action must match global evidence. Lancet. 2010 Nov 13;376(9753):1619-21.
10. Ministry of Public Health (CU). [Statistical Yearbook. Cuba] [Internet]. Havana: Ministry of Public Health (CU); 2009 [cited 2010 Aug 24]. 243 p. Available from: http://files.sld.cu/dne/ files/2010/04/anuario-2009e3.pdf. Spanish.

11. PAHO. Nota Estratégica. Desarrollo de la Cooperación Técnica de la OMS/OPS con la República de Cuba, 2008-2010 [Internet]. Havana: Pan American Health Organization; 2008 [cited 2008 Mar 28]. Available from: http://www.paho.org/spanish/d/csu/CCS -CubaNotaEstrategica.pdf. Spanish.

12. Prince $M$, Acosta $D$, Chiu $H$, Scazufca $M$, Varghese M; 10/66 Dementia Research Group Dementia diagnosis in developing countries: a cross-cultural validation study. Lancet. 2003 Mar 15;361(9361):909-17.

13. Prince $M$, Ferri CP, Acosta D, Albanese $E$, Llibre J, Guerra M. The Protocols for the 10/66 
Dementia Research Group Population-Based Research Programme. BMC Public Health. 2007 Jul 20;7:165-9.

14. Llibre Rodriguez JJ, Ferri CP, Acosta D, Guerra $M$, Huang $Y$, Jacob KS, et al. Prevalence of dementia in Latin America, India, and China: a population-based cross-sectional survey. Lancet. 2008 Aug 9;372(9637):464-74.

15. Whitworth JA; World Health Organization, International Society of Hypertension of Hypertension Group. 2003 World Health Organization (WHO)/ International Society of Hypertension (ISH) statement on management of hypertension. J Hypertens. 2003 Nov;21(11):1983-92.

16. Copeland JR, Dewey ME, Griffiths-Jones HM. A computerized psychiatric diagnostic system and case nomenclature for elderly subjects: GMS and AGECAT. Psychol Med. 1986 Feb;16(1):89-99.

17. Hall KS, Hendrie HC, Brittain HM, Norton JA, Rodgers DD, Prince CS, et al. The development of a dementia screening interview in two distinct languages. Int $\mathrm{J}$ Methods Psychiatr Res. 1993;3:1-28.

18. Ganguli M, Chandra V, Gilbey JE, Ratcliff G, Sharma SD, Pandav R, et al. Cognitive test performance in a community-based non demented elderly sample in rural India: the Indo-US Cross-National Dementia Epidemiology Study. Int Psychogeriatr. 1996 Winter;8(4):507-24.

19. Dewey ME, Copeland JR. Diagnosis of dementia from the history and aetiology schedule. Int $J$ Geriatr Psychiatry. 2001 Sep;16(9):912-7.

20. American Psychiatric Association. Diagnostic and Statistical Manual of Mental Disorders DSMIV. 4th ed. Washington DC: American Psychiatric Association; 1994 Jan 15. 886 p.

21. Hatano S. Experience from a multicentre stroke register: a preliminary report. Bull World Health Organ. 1976;54(5):541-53.

22. Broe GA, Akhtar AJ, Andrews GR, Caird FI, Gilmore AJ, McLennan WJ. Neurological disorders in the elderly at home. J Neurol Neurosurg Psychiatry. 1976 Apr;39(4):362-6.

23. Special Report from the National Institute of Neurological Disorders and Stroke. Classification of cerebrovascular disease III. Stroke. 1990;21:637-76.

24. American Diabetes Association. Standards of Medical Care in Diabetes-2010. Diabetes Care 2010 Jan;33 Suppl 1:S11-61.

25. Third report of the National Cholestero Education Program (NCEP) Expert Panel on the detection, evaluation, and treatment of high blood cholesterol in adults (Adult Treatment panel III). Executive summary. Bethesda: National Institutes of Health (US); 2001 May.

26. Rehm J, Üstün TB, Saxena S, Nelson CB, Chatterji $S$, Ivis $F$, et al. On the development and psychometric testing of the WHO screening instrument to assess disablement in the general population. Int J Methods Psychiatr Res. 1999 Jun;8(2):110-22.

27. Sousa RM, Dewey ME, Acosta D, Jotheeswaran AT, Castro-Acosta E, Ferri CP, et al. Measuring disability across cultures - the psychometric properties of the WHODAS II in older people from seven low- and middle-income countries. The 10/66 Dementia Research Group populationbased survey. Int J Methods Psychiatr Res. 2010 Mar;19(1):1-17.

28. Gilleard CJ, Belford H, Gilleard E, Whittick JE, Gledhill K. Emotional distress amongst the supporters of the elderly mentally infirm. $\mathrm{Br} \mathrm{J}$ Psychiatry. 1984 Aug;145:172-7.

29. Davis KL, Marin DB, Kane R, Patrick D, Peskind ER, Raskind MA, et al. The Caregiver Activity Survey (CAS): development and validation of a new measure for caregivers of persons with Alzheimer's disease. Int J Geriatr Psychiatry. 1997 Oct;12(10):978-88.

30. Barros AJ, Hirakata VN. Alternatives for logistic regression in cross-sectional studies: an empirical comparison of models that directly estimate the prevalence ratio. BMC Med Res Methodol. 2003 Oct 20;3:21.

31. Ostchega Y, Dillon CF, Hughes JP, Carroll M, Yoon S. Trends in hypertension prevalence, awareness, treatment, and control in older U.S. adults: data from the National Health and Nutrition Examination Survey 1988 to 2004 . J Am Geriatr Soc. 2007 Jul:55(7):1056-65.

32. National Institutes of Health (US); National Heart, Lung and Blood Institute (US), editors. Morbidity and Mortality: 2009 Chart Book on Cardiovascular, Lung, and Blood Diseases. Bethesda: National Institutes of Health (US); 2009. 114 p.

33. Lobo A, Launer LJ, Fratiglioni L, Andersen K, Di Carlo A, Breteler MM, et al. Prevalence of dementia and major subtypes in Europe: A collaborative study of population-based cohorts. Neurologic Diseases in the Elderly Research Group. Neurology. 2000;54(11 Suppl 5):S4-9.

34. StataCorp 2007. Stata Statistical Software: Release 10. College Station TX: Statcorp LP.

35. Salud, Bienestar y Envejecimiento en las Américas. SABE. Resumen Ejecutivo. Havana: Ministry of Public Health (CU); 2000 Oct. 64 p. Spanish.

36. Longo-Mbenza B, Ngoma DV, Nahimana D, Mayuku DM, Fuele SM, Ekwanzala F, et al. Screen detection and the WHO stepwise approach to the prevalence and risk factors of arterial hypertension in Kinshasa. Eur $J$ Cardiovasc Prev Rehabil. 2008 Oct;15(5):503-8.

37. Gu D, Reynolds K, Wu X, Chen J, Duan X, Muntner $\mathrm{P}$, et al. Prevalence, Awareness, Treatment, and Control of Hypertension in China. Hypertension. 2002 Dec;40(6):920-7.

38. Sanderson S. Hypertension in the elderly: pressure to treat? Health Trends. 1996;28(4):117-21.

39. Cowie CC, Rust KF, Byrd-Holt DD, Eberhardt MS, Flegal KM, Engelgau MM, et al. Prevalence of diabetes and impaired fasting glucose in adults in the U.S. population: National Health And Nutrition Examination Survey 1999-2002. Diabetes Care. 2006 Jun;29(6):1263-8.

40. Disorders of the cardiovascular system. Vascular disease. In: Kasper DL, Braunwald E, Fauci AS, Hauser SL, Longo DL, Jameson JL, et al, editors. Harrison's Principles of Internal Medicine. 16th ed. New York: McGraw-Hill Professional; c2005. Part 8, Section 1; p 1425-86.

41. Pérez-Sempere A. Morbilidad por enfermedad cerebrovascular en España: incidencia y prevalencia. Rev Neurol. 1999 Nov 1-15;29(9):879-81. Spanish.

42. Saposnik G, Brutto OH; Iberoamerican Society of Cerebrovascular Diseases. Stroke in South America. A systematic review of incidence, prevalence, and stroke subtypes. Stroke. 2003 Sep;34(9):2103-7.

43. Llibre Rodríguez JJ, Valhuerdi A, Fernández O, Llibre JC, Porto R, López AM, et al. Prevalence of Stroke and Associated Risk Factors in Older Adults in Havana City and Matanzas Provinces, Cuba (10/66 Population-Based Study). MEDICC Rev. 2010 Jul;12(3):20-6.

44. Llibre Rodríguez JJ, Valhuerdi A, Sánchez II, Reyna C, Guerra MA, Copeland JR, et al. The Prevalence, Correlates and Impact of Dementia in Cuba. A 10/66 Group population-based survey. Neuroepidemiology. 2008;31(4):243-51.

45. Ferri CP, Prince $M$, Brayne $C$, Brodaty $H$, Fratiglioni L, Ganguli M, et al. Global prevalence of dementia: a Delphi consensus study. Lancet. 2005 Dec 17;366(9503):2112-7.

46. Alzheimer's Disease International. World Alzheimer Report 2009. London: Alzheimer's Disease International; 2009.

47. Prince M, Llibre Rodriguez JJ, Noriega L, López A, Acosta D, Albanese E, et al. The 10/66 Dementia Research Group's fully operationalised DSM IV dementia computerized diagnostic algorithm, compared with the 10/66 dementia algorithm and a clinician diagnosis: a population validation study. BMC Public Health. 2008 Jun 24;8:219.

48. Llibre Rodríguez JJ, Fernández $\mathrm{Y}$, Marcheco $B$, Contreras N, López AM, Otero $M$, et al Prevalence of Dementia and Alzheimer's Disease in a Havana Municipality: A CommunityBased Study among Elderly Residents. MEDICC Rev. 2009 Apr;11(2):29-35.

49. Schargrodsky $H$, Hernández-Hernández $R$ Champagne BM, Silva $H$, Vinueza $R$, Silva Ayçaguer LC, et al. CARMELA: assessment of cardiovascular risk in seven Latin American cities. Am J Med. 2008 Jan;121(1):58-65.

\section{THE AUTHORS}

Juan de Jesús Llibre Rodríguez (Corresponding author: mguerra@infomed.sld.cu), physician specializing in internal medicine. Full professor, senior researcher and director, Alzheimer Studies Center, Finlay-Albarrán Medical Faculty of the Medical University of Havana, Cuba.

Adolfo Valhuerdi Cepero, physician specializing in internal medicine, with a master's degree in urgent care. Assistant professor, Faustino Pérez Provincial University Hospital, Matanzas, Cuba.

Marina Calvo Rodríguez, psychiatrist with a master's degree in health psychology. Instructor, La Lisa Community Mental Health Center, Havana, Cuba.

Rosa M. García Klibanski, psychiatrist with a master's degree in social psychiatry, 14 de Junio University Polyclinic, Luyano, Havana, Cuba.

Milagros Guerra Hernández, psychiatrist with a masters in longevity studies. Instructor, 27 de Noviembre University Polyclinic, Marianao, Havana, Cuba.

Tania Laucerique Pardo, physician specializing in internal medicine with a master's degree in geriatrics and gerontology. Associate professor, Carlos J. Finlay Hospital, Havana, Cuba.

Ana M. López Medina, physician specializing in family medicine, geriatrics and gerontology, with a master's degree in longevity studies. Instructor, Héroes del Corynthia Polyclinic, Havana, Cuba.

Juan Carlos Llibre Guerra, physician specializing in family medicine, with a master's degree in longevity studies. Neurology resident and instructor, Cuban Neurology and Neurosurgery Institute, Havana, Cuba.

Lisseth Noriega Fernández, psychiatrist, Marianao Community Mental Health Center, Havana, Cuba. 
Isis Y. Sánchez Gil, psychiatrist with a master's degree in longevity studies. Associate professor, Ana Betancourt Polyclinic, Playa, Havana, Cuba.

Rudbeskia Porto Alvarez, physician specializing in family medicine, with a master's degree in urgent care. Instructor, José R. López Tabranes Hospital, Matanzas, Cuba.
Francis Arencibia Márquez, psychologist with a master's degree in longevity studies. Assistant professor, Faustino Pérez Provincial University Hospital, Matanzas, Cuba.

Beatriz Marcheco Teruel, physician specializing in clinical genetics with a doctorate in medical sciences. Full professor and director, National Medical Genetics Center, Havana, Cuba.
Carmen Moreno Carbonell, physician specializing in epidemiology. Full professor, Finlay-Albarrán Medical Faculty of the Medical University of Havana, Cuba.

Submitted: January 2, 2011

Approved for publication: October 12, 2011

Disclosures: None

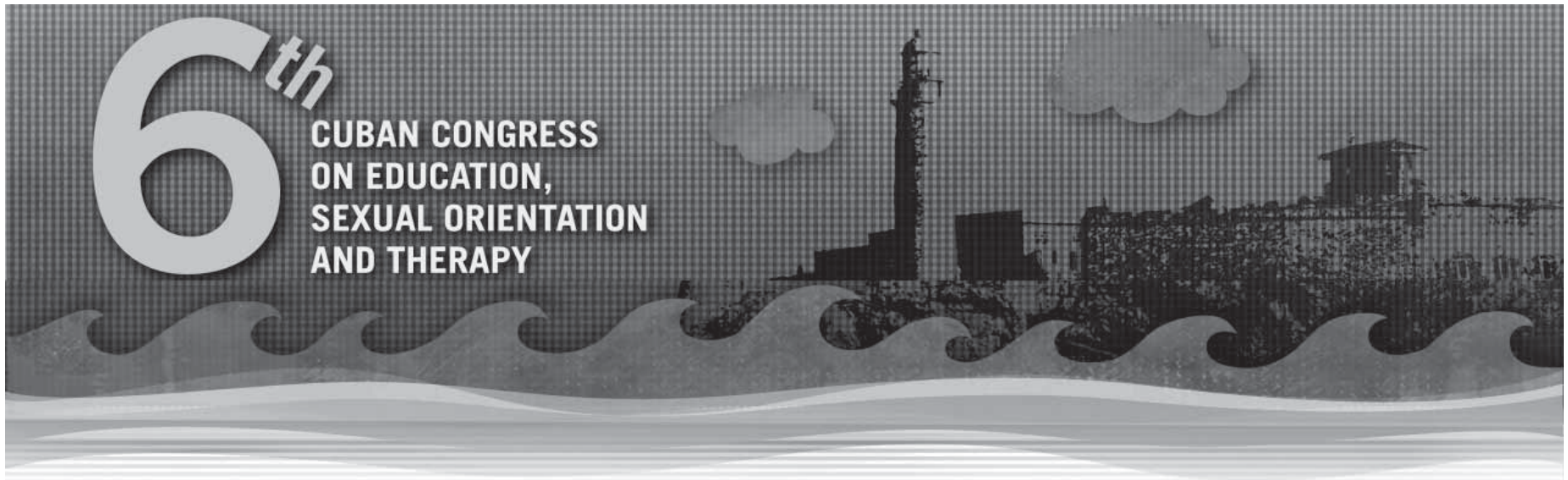

\section{SEX EDUCATION IN SOCIAL} TRANSFORMATION PROCESSES
VI Cuban Congress on Education, Sexual Orientation and Therapy 23-26 January 2012

Palacio de Convenciones, Havana, Cuba

Congress Chair

M.Sc. Mariela Castro Espín

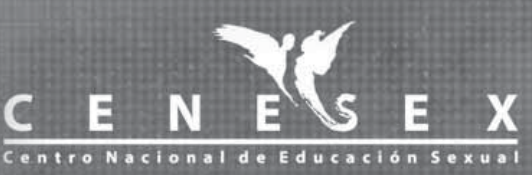

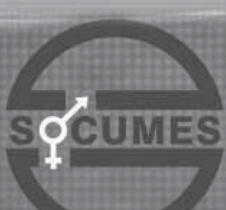

More information:

http://www.sexologiacuba.com - http://www.cenesexualidad.sld.cu

(j) ECNSCS 